HIP-2011-19/TH

June 16, 2021

\title{
Mass spectrum and thermodynamics of quasi-conformal gauge theories from gauge/gravity duality
}

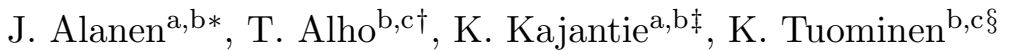 \\ ${ }^{a}$ Department of Physics, P.O.Box 64, FI-00014 University of Helsinki, Finland \\ ${ }^{\mathrm{b}}$ Helsinki Institute of Physics, P.O.Box 64, FI-00014 University of Helsinki, Finland \\ ${ }^{\mathrm{c}}$ Department of Physics, University of Jyväskylä
}

\begin{abstract}
We use gauge/gravity duality to study simultaneously the mass spectrum and the thermodynamics of a generic quasi-conformal gauge theory, specified by its beta function. The beta function of a quasi-conformal theory almost vanishes, and the coupling is almost constant between two widely separated energy scales. Depending on whether the gravity dual has a black hole or not, the mass spectrum is either a spectrum of quasinormal oscillations or a normal $T=0$ mass spectrum. The mass spectrum is quantitatively correlated with the thermal properties of the system. As the theory approaches conformality, the masses have to vanish. We show that in this limit, the masses calculated via gauge/gravity duality satisfy expected scaling properties.
\end{abstract}

*janne.alanen@helsinki.fi

${ }^{\dagger}$ timo.s.alho@jyu.fi

${ }^{\ddagger}$ keijo.kajantie@helsinki.fi

${ }^{\S}$ kimmo.i.tuominen@jyu.fi 


\section{Introduction}

In this article, we shall consider gravity dilaton -systems dual to quasi-conformal gauge theories. Their characteristic feature is the presence of two widely different energy scales, $\Lambda_{\mathrm{ETC}}$ and $\Lambda_{\mathrm{TC}}$, between which the coupling constant of the theory evolves very slowly; the theory is almost conformal, the beta function almost vanishes. Below $\Lambda_{\mathrm{TC}}$ and above $\Lambda_{\mathrm{ETC}}$ the coupling runs similarly to asymptotically free theories.

Quasi-conformal gauge theories are of current interest for beyond the Standard Model physics in the context of walking technicolor [1]. Our model lacks proper flavor dynamics of technicolor, but this connection nevertheless motivates our notation and the quantitative estimate $\Lambda_{\mathrm{ETC}} \sim 10^{3} \Lambda_{\mathrm{TC}}$.

Our gravity dual, Eq. 2.2 below, is based on the improved holographic QCD presented in [2, 3, 4] and further developed in [5, 6, 7]. The extension of the model from QCD to technicolor is based on building the required physics in the ansatz for the beta function of the coupling $g^{2}$ of the theory, in a certain scheme. For this we use the ansatz

$$
\beta(\lambda)=-c \lambda^{2} \frac{(1-\lambda)^{2}+e}{1+a \lambda^{3}}, \quad \lambda=N_{c} g^{2},
$$

which near $\lambda=0,1$ and $\infty$ can be expanded or approximated as

$$
\begin{aligned}
\beta(\lambda) & =-c(1+e) \lambda^{2}+2 c \lambda^{3}-c \lambda^{4}+\mathcal{O}\left(\lambda^{5}\right), \\
& \approx-\frac{c}{1+a}\left[(1-\lambda)^{2}+e\right], \quad \lambda \text { near } 1, \\
& =-\frac{c}{a} \lambda+\frac{2 c}{a}-\frac{c(1+e)}{a} \frac{1}{\lambda}+\mathcal{O}\left(\frac{1}{\lambda^{2}}\right) .
\end{aligned}
$$

In these one has built asymptotic freedom in the ultraviolet (UV) domain, i.e., as $\lambda \rightarrow 0$, walking near $\lambda=1$ provided $e$ is small and confinement in the infrared (IR) large $\lambda$ domain if $a=2 c / 3$ [2]. The values of parameters $c, a, e$ reflect the physics one wants to describe: $c \gtrsim 10$ fixes the high $T$ phase transition to be of first order [6] and $e$ has to be a small number to describe near conformality. The case $e=0$ leads to an infrared fixed point (IRFP) [7].

Thermodynamics of the model has already been studied in [6]: the system can exist in three different phases with phase transitions at $T=T_{\mathrm{TC}} \approx \Lambda_{\mathrm{TC}}$ and $T=T_{\mathrm{ETC}} \approx \Lambda_{\mathrm{ETC}}$. The main goal of this article is to study the spectrum of the theory, which is either a usual $T=0$ mass spectrum, if there is no black hole in the gravity dual or a quasinormal spectrum [8] with complex energies, if there is one. The relation between the numerical values of $T_{\mathrm{TC}}, T_{\mathrm{ETC}}$ and the mass values is then also obtained.

Of particular interest will be the behavior of the mass spectrum when conformality is approached, $e \rightarrow 0$. We shall show that in this limit the mass spectrum as computed from gauge/gravity duality satisfies Miransky scaling [9]

$$
m(c, e)=A(c, e) \exp \left[-\left(\frac{2}{3}+\frac{1}{c}\right) \frac{\pi}{\sqrt{e}}\right]
$$

where $A(c, e)$ is slowly varying when $e \rightarrow 0$. 
The bottom-up gravity dual presented here lacks detailed flavor dynamics and is thus not intended to be holographic description of walking technicolor. Examples of top down technicolor duals are given in [10, 11]. In any case its merit is that it presents a concrete gravitational dual with phase transitions at two widely separated energy scales. A general discussion of these in terms of multiple big black holes is given in Section G of [3] .

Section 2 discusses the gravity dual and, in particular, the determination of the dilaton potential which, after solving the Einstein equations, leads to the beta function (1.1). Details of numerical evaluation of the background are also presented. Section 3 reviews the thermodynamics, already discussed in [6]. Depending on parameters, the model can describe either first order or continuous transitions. General principles of determining the masses and quasinormal modes from Green's functions are summarised in Section 4. One practical limitation of the present dual is that it can only be computed numerically. However, one can present a simplified version of the model containing qualitatively its essential properties [20], this is applied to the determination of the quasinormal modes in Section 5. The full computation of the spectrum is carried out in Section 6 .

\section{Gravity dual}

\subsection{Equations}

The gravity equations of the model are as follows [3, 6]. The gravity action, including the scalar field $\phi(z)$, in standard notation and in the Einstein frame, is

$$
S=\frac{1}{16 \pi G_{5}} \int d^{5} x \sqrt{-g}\left[R-\frac{4}{3}\left(\partial_{\mu} \phi\right)^{2}+V(\phi)\right] .
$$

With the metric ansatz

$$
d s^{2}=b^{2}(z)\left[-f(z) d t^{2}+d \mathbf{x}^{2}+\frac{d z^{2}}{f(z)}\right],
$$

the three functions $b(z), f(z)$ in the metric and the scalar field $\phi(z)$ are determined from the three equations $\left(\dot{b} \equiv b^{\prime}(z)\right.$, etc. $)$

$$
\begin{aligned}
& 6 \frac{\dot{b}^{2}}{b^{2}}+3 \frac{\ddot{b}}{b}+3 \frac{\dot{b}}{b} \frac{\dot{f}}{f}=\frac{b^{2}}{f} V(\phi), \\
& 6 \frac{\dot{b}^{2}}{b^{2}}-3 \frac{\ddot{b}}{b}=\frac{4}{3} \dot{\phi}^{2}, \\
& \frac{\ddot{f}}{\dot{f}}+3 \frac{\dot{b}}{b}=0 .
\end{aligned}
$$

Further, from the functions so evaluated, the beta function follows as

$$
\beta(\lambda)=b \frac{d \lambda}{d b}=b \frac{\dot{\lambda}}{\dot{b}}, \quad \lambda(z)=e^{\phi(z)} \sim g^{2} N_{c}
$$


Thus $\lambda=e^{\phi(z)}$ is the coupling at the energy scale $b(z)$. Often it is practical to use the rescaled form

$$
X \equiv \frac{\beta(\lambda)}{3 \lambda}
$$

and to define

$$
W=-\frac{\dot{b}}{b^{2}}, \quad b \dot{W}=2 \frac{\dot{b}^{2}}{b^{2}}-\frac{\ddot{b}}{b},
$$

which changes 2.4 to first order form. Inserting further $\dot{W}=W^{\prime}(\lambda) \dot{\lambda}$ and then $\dot{\lambda}=\dot{b} \beta / b=$ $-b W \beta$ permits one to solve $W(\lambda)$ in terms of $\beta$ (Eq. (2.11) below).

Note the two roles, mathematical and physical, played by $\beta(\lambda)$. Irrespective of the physical context this combination of $\lambda, b$, arises very naturally in the numerical solution of (2.3)-(2.5) (see, e.g., (2.9) or (2.10) below). Physically it models the running of the coupling $\lambda(z)$ as a function of the energy scale $\sim b(z)$.

Note further that $\beta(\lambda)$ can be solved from $(2.6)$ only after all the fields have been solved. This requires a knowledge of $V(\lambda)$. However, given $\beta(\lambda)$ one can from $(2.3)$ with $f=1$ solve

$$
V_{0}(\lambda)=12 W^{2}(\lambda)\left[1-\left(\frac{\beta}{3 \lambda}\right)^{2}\right], \quad f(z)=1 .
$$

Here $W(\lambda)$ can be solved in terms of the beta function 2.6 and, introducing the explicit ansatz (1.1), one gets

$$
\begin{aligned}
& \frac{W(\lambda)}{W(0)}=\exp \left[-\frac{4}{9} \int_{0}^{\lambda} d \bar{\lambda} \frac{\beta(\bar{\lambda})}{\bar{\lambda}^{2}}\right] \\
&=\exp \left\{\frac { 2 c } { 2 7 a } \left[2 \sqrt{3} a^{1 / 3}\left(-2+a^{1 / 3}(1+e)\right)\left(\arctan \left[\frac{-1+2 a^{1 / 3} \lambda}{\sqrt{3}}\right]-\arctan \frac{-1}{\sqrt{3}}\right)+\right.\right. \\
&\left.\left.\left.a^{1 / 3}\left(2+a^{1 / 3}(1+e)\right) \log \left[\left(1+a^{1 / 3} \lambda\right)^{2} /\left(1-a^{1 / 3} \lambda+a^{2 / 3} \lambda^{2}\right)\right]+2 \log \left[1+a \lambda^{3}\right]\right)\right]\right\}
\end{aligned}
$$

where $W(0)$ is the inverse of the AdS radius:

$$
W(0)=\frac{1}{\mathcal{L}}
$$

At large $\lambda$, Eq. 2.11) implies that $W(\lambda) \sim \lambda^{4 c /(9 a)}$ so that $V_{0}(\lambda) \sim \lambda^{8 c /(9 a)}$, more detailed asymptotic expressions are given in [20] and below in Eqs. 22.23 - 2.28). As shown in [2], confinement requires that the large $\lambda$ limit be $V \sim \lambda^{4 / 3}(\log \lambda)^{(\alpha-1) / \alpha}$ with $\alpha>1$. Thus we fix

$$
a=\frac{2}{3} c .
$$

A mass spectrum of type $m^{2} \sim n$ [12] further implies $\alpha=2$. This is also built in Eq. (2.27) below. To avoid spoiling the UV small- $z$, small- $\lambda$ behavior we shall use the potential

$$
V(\lambda)=V_{0}(\lambda) \sqrt{\frac{\log \left(F+\lambda^{4}\right)}{\log F}} .
$$


Here $F$ is a parameter which sets the scale at which confinement effects set in. Since the quasi-conformal effects operate, by construction, at $\lambda \approx 1$ we certainly expect $F^{1 / 4} \gg 1$ and choose $F=1000$, to have a large separation between ETC and TC scales. For comparison, in [4] the potential for $\mathrm{SU}\left(N_{c}\right)$ Yang-Mills thermodynamics was chosen to be

$$
V_{\mathrm{IHQCD}}(\lambda)=\frac{12}{\mathcal{L}^{2}}\left\{1+0.04128 \lambda+(7.35 \lambda)^{4 / 3} \sqrt{\log \left[1+(13 \lambda)^{2}\right]}\right\} .
$$

We re-emphasize that $V(\lambda)$ in (2.14) is given in terms of a quantity $\beta(\lambda)$ which one knows only after one has solved the problem. So one has to numerically check that the output beta function to sufficient accuracy reproduces the input one; this seems to work very well [6].

\subsection{Numerical solution}

The numerical solution of Eqs. (2.3) - 2.5) with $V$ given by (2.14), means finding a set of functions $b(z), f(z), \lambda(z)$ parametrised by the horizon value $\lambda_{h}=\lambda\left(z_{h}\right), f\left(z_{h}\right)=0$ [4, 6] . Various physical quantities computed from the set of functions are then functions of $\lambda_{h}$. For example, see Fig. 2 for $T=T\left(\lambda_{h}\right)$.

To solve Eqs. 2.3) - 2.5 it is practical to use the $W$ in 2.8 to write them in the form

$$
\begin{aligned}
\dot{W} & =4 b W^{2}-\frac{1}{f}\left(W \dot{f}+\frac{1}{3} b V\right), \\
\dot{b} & =-b^{2} W, \\
\dot{\lambda} & =\frac{3}{2} \lambda \sqrt{b \dot{W}}, \\
\ddot{f} & =3 \dot{f} b W,
\end{aligned}
$$

Details of numerical integration can be found in Sect. 2.2 of [6]. We add here a number of points relevant for the purposes of this article.

1. The choice of the set of $\lambda_{h}$ values depends on whether one studies thermodynamics or the mass spectrum. In the former case one has to integrate over a large range of temperatures so that one chooses a large set of $\lambda_{h}$ values from small values, $\lesssim 1$ for the UV and $\gg 1$ for the IR. For the mass spectrum one should find a special no-black hole solution $f(z)=1$. How this is to be done is discussed in Appendix A. However, one can also possible to start from a $f \neq 1$ numerical solution with one very large value of $\lambda_{h}$ and correspondingly very large value of $z_{h}$. Staying within the values of $0<z<z_{h}$, for which $f(z)$ is very close to unity, one has an approximate solution of $b(z), \lambda(z)$ for $f=1$.

2. The initial condition $b_{h}=b\left(z_{h}\right)$ follows from $b\left(z_{h}\right)=\exp \left[\int^{\lambda_{h}} d \lambda / \beta(\lambda)\right]$ :

$$
\begin{aligned}
c(1+e) \log b_{h}= & \frac{1}{\lambda_{h}}-\frac{2}{1+e} \log \left[c(1+e) \lambda_{h}\right] \\
& +\frac{1-e+a(1+e)^{2}}{\sqrt{e}(1+e)}\left(\arctan \frac{1-\lambda_{h}}{\sqrt{e}}-\arctan \frac{1}{\sqrt{e}}\right) \\
& -\frac{a(1+e)^{2}-2}{2(1+e)} \log \frac{\left(1-\lambda_{h}\right)^{2}+e}{1+e},
\end{aligned}
$$


where the constant of integration is fixed so that the leading UV $\lambda \rightarrow 0$ behavior is the 2-loop one, i.e., the two last terms in 2.20 vanish at $\lambda_{h}=0$.

3. The initial condition $W_{h}=b_{h} V\left(\lambda_{h}\right) /\left(-3 \dot{f}_{h}\right)$ follows from 2.3 by requiring that all fields remain finite at the horizon. For $\dot{f}_{h}$ one can take an arbitrary negative value. The energy unit is determined so that at $z \rightarrow 0$

$$
b_{0} \lambda(z)=\frac{1}{\log [1 /(\Lambda z)]}-\frac{b_{1}}{b_{0}^{2}} \frac{\log \log [1 /(\Lambda z)]}{\log ^{2}[1 /(\Lambda z)]}, \quad b_{0}=c(1+e), \quad b_{1}=-2 c
$$

where we choose units $\Lambda=1$.

4. Due to large ETC and TC scale separation large values of $z$ enter into the analysis and it is useful to have an analytic approximation to numerics. One cannot analytically solve the equations at large $z$ and large $\lambda$, but one finds that a good approximation for $b(z)$ is 1

$$
b(z)=b_{0} e^{-(\Xi z)^{2}} .
$$

$\Xi$ here is an IR scale, analogous to the $\Lambda$ in the UV. Another definition will be given below in 6.2 . From the numerical solutions we can determine $\Xi$, and we find that typically $\Xi / \Lambda \sim 0.001$. This is consistent with the large energy scale splitting $\mathrm{ETC} / \mathrm{TC} \sim 1000$. Inserting this to the equations of motion above one finds that, up to corrections $1+\mathcal{O}\left(1 / z^{2}\right) \sim 1+\mathcal{O}(1 / \log \lambda)$,

$$
\begin{aligned}
b(z) & =b_{0} e^{-(\Xi z)^{2}}, \\
W(z) & =\frac{2}{\mathcal{L}} e^{(\Xi z)^{2}}(\Xi z), \quad \mathcal{L}=\frac{b_{0}}{\Lambda}, \\
\bar{\lambda}(z) & =e^{3(\Xi z)^{2} / 2}(\Xi z)^{3 / 4}, \Xi z=\left(\frac{2}{3} \log \bar{\lambda}\right)^{1 / 2}, \\
\beta(\lambda) & =-\frac{3}{2} \lambda\left(1+\frac{1}{4(\Xi z)^{2}}+\mathcal{O}\left(\frac{1}{z^{4}}\right)\right), \\
V(z) & =9 W^{2}(z)=\frac{36}{\mathcal{L}^{2}} e^{2(\Xi z)^{2}}(\Xi z)^{2}=\frac{36}{\mathcal{L}^{2}} \bar{\lambda}^{4 / 3}\left(\frac{2}{3} \log \bar{\lambda}\right)^{1 / 2}, \\
f(z) & =1-\frac{z_{h}}{z} e^{3 \Xi^{2}\left(z^{2}-z_{h}^{2}\right)}, \\
-\dot{f}\left(z_{h}\right) & =4 \pi T=6 \Xi^{2} z_{h} .
\end{aligned}
$$

Here $\bar{\lambda} \equiv \lambda / \lambda_{0}$, where $\lambda_{0}$ is the constant of integration. These equations will in practice (Appendix A) be important as the initial conditions for computing the $f=1$ gravity solutions.

5. As a concrete example of computed bulk fields, Fig. 1 shows $z b(z), \lambda(z), W(z)$ for one set of parameters; $\lambda_{h}=10^{6}$ with $z_{h}=1805$ was used here. This also shows how the required structure with two widely separated energy scales is reflected in the bulk fields. For example, the coupling $\lambda(z)$ first approaches an almost stable IR fixed

\footnotetext{
${ }^{1}$ Note that one may use $z b(z)=b_{0} \exp \left[-(\Xi z)^{2}\right]$ to capture both the UV and IR behavior, as in the model of Section 5 [20. For this discussion of the behavior deep in the IR, it suffices to take only the Gaussian tail into account. Formulas for a more general parametrisation $b \sim \exp \left[-(\Xi z)^{\alpha}\right] z^{p}$ are given in $[20$.
} 

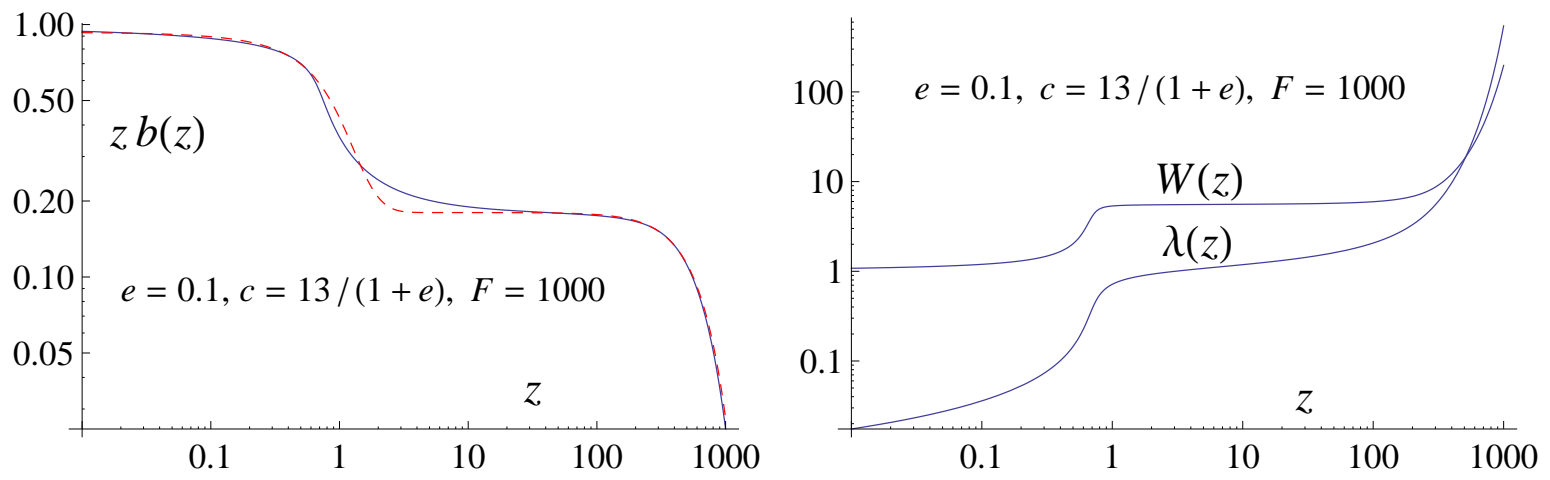

Figure 1: The functions $z b(z), \lambda(z), W(z)$ for $e=0.1, c=13 /(1+e), F=1000$. The dashed curve is the fit 2.29) with parameters given in the text. The horizon is at $z_{h}=1805$ and at this point $\lambda(z)$ has grown to the value $10^{6}$.

point $\lambda=1$ near $z=1$. However, instead of freezing at this value, $\lambda$ gradually starts growing and ultimately at large $z$ grows as specified by equation 2.25 valid at large $z: \lambda \sim \exp \left(\frac{3}{2} \Xi^{2} z^{2}\right), W \sim \exp \left(\Xi^{2} z^{2}\right)$. The transition between a UV fixed point and a quasi stable IR fixed point is particularly well seen in the behavior of $b(z)$, which first behaves as $\mathcal{L}_{U} / z$, then as $\mathcal{L}_{I} / z$ with a decreasing AdS radius and ultimately in the far IR shows the gaussian decrease 2.23). In fact, the numerically computed $z b(z)$ in Fig. 1 can be fitted by an expression of the type

$$
z b(z)=\left[\mathcal{L}_{U}-\left(\mathcal{L}_{U}-\mathcal{L}_{I}\right) \Theta\left(z-z_{\text {qir }}\right)\right] e^{-\Xi^{2} z^{2}}
$$

where in Fig. $11 \mathcal{L}_{U}=1.1, \mathcal{L}_{I}=0.18, \Theta\left(z-z_{\text {qir }}\right)=\frac{1}{2}+\frac{1}{2} \tanh ((z-0.6) / 0.8), \Xi=1 / 730$.

\section{Thermodynamics}

The thermodynamics of the present model has been discussed in [6], but with a different functional dependence on the parameter $F$ in the dilaton potential. More precisely, in [6] the $F$-dependent term in (2.14) was chosen to approach one as $e \rightarrow 0$, while now the confinement factor in 2.14 is independent of the walking parameter $e$. This is more realistic since confinement enters at large $\lambda$, far from the IR fixed point $\lambda=1$.

To summarize the results, we shall choose as reference values

$$
e=0.1, c(1+e)=13, F=1000 .
$$

The combination $c(1+e)$ is the leading 1loop coefficient of the beta function (1.1). One then generates numerically a family of solutions $b(z), \lambda(z), f(z), f\left(z_{h}\right)=0$, parametrised by the value $\lambda_{h}=\lambda\left(z_{h}\right)$, and normalises the solutions as in 2.21). From the solutions one then 


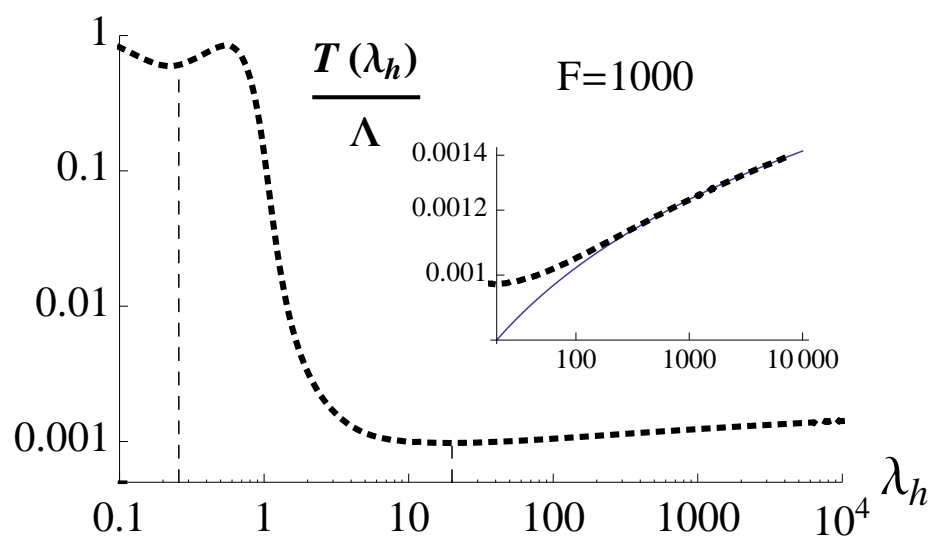

Figure 2: The function $T\left(\lambda_{h}\right)$ obtained for $e=0.1, c=13 /(1+e), F=1000$. The first minimum is at $\lambda_{h}=0.218, T / \Lambda=0.8$ the second at $\lambda_{h}=20, T / \Lambda=0.001$. The corresponding transitions are at $T / \Lambda=0.653$ (Fig. 3) and at $T / \Lambda=0.00098$ (Fig. 4). The behavior at large $\lambda_{h}$ (inset) can be fitted by $0.00050 \log \lambda_{h}^{0.47}$.

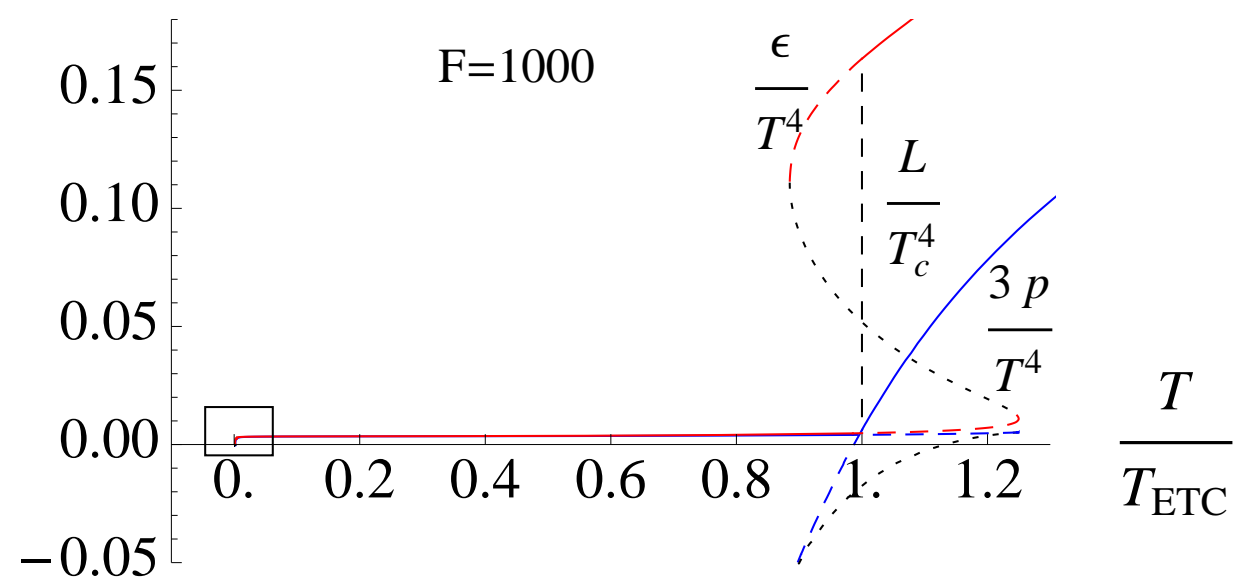

Figure 3: The equation of state obtained for $e=0.1, c=13 /(1+e), F=1000$ for the region around the first order ETC phase transition at $T_{\mathrm{ETC}}=0.602 \Lambda$. Dotted lines are unstable, dashed lines metastable (supercooled or -heated). The boxed confinement transition to the low $T$ phase with $p=0$ at $T_{\mathrm{TC}}=0.000977 \Lambda$ is blown up in Fig. 4 .

computes thermodynamics by evaluating

$$
\begin{aligned}
4 \pi T & =-\dot{f}\left(z_{h}\right), \quad s=\frac{b^{3}\left(z_{h}\right)}{4 G_{5}} \\
p & =\frac{1}{4 G_{5}} \int_{\bar{\lambda}_{h}}^{\infty} d \bar{\lambda}_{h}\left(-\frac{d T}{d \bar{\lambda}}\right) b^{3}\left(\bar{\lambda}_{h}\right) \\
\epsilon & =T s-p, \\
c_{s}^{2} & =\frac{s(T)}{T s^{\prime}(T)}=\frac{\beta(\lambda)}{3 T} \frac{d T}{d \lambda_{h}}=\beta(\lambda) \frac{d}{d \lambda_{h}} \ln T^{1 / 3}
\end{aligned}
$$




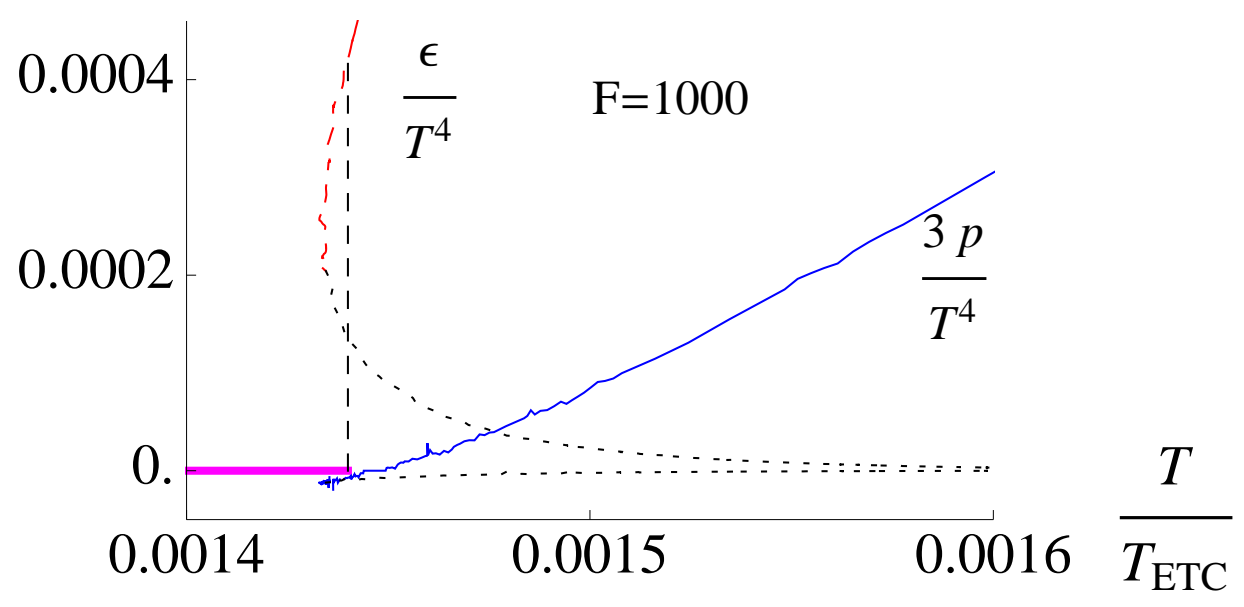

Figure 4: The equation of state obtained for $e=0.1, c=13 /(1+e), F=1000$ in the region around the confinement transition to a low $T$ phase with $p=0$ (thick red line) at $T_{\mathrm{TC}}=0.000977 \Lambda$. Dotted lines are unstable, dashed lines metastable (supercooled or -heated).

Each quantity can be regarded as a function of $z_{h}$ or $\lambda_{h}$ or $T$. If the extreme UV pressure is $g_{\text {eff }} \pi^{2} T^{4} / 90$, then

$$
\frac{\mathcal{L}^{3}}{4 G_{5}}=\frac{2}{45 \pi} g_{\text {eff }}
$$

Thermal physics is basically contained in the function $T\left(\lambda_{h}\right)$, shown in Fig. 2, The model is constructed so as to have two transitions and, accordingly, there are two minima. The mechanically stable regions with $c_{s}^{2}>0$ correspond to $d T / d \lambda_{h}<0$ (cf. Eq. (3.5) and remember that $\beta<0)$. With the structure of Fig. 2 one can have $T$ equal in two different decreasing branches of $T\left(\lambda_{h}\right)$, and if they also have the same pressure, one has two different phases in thermal and mechanical equilibrium, i.e. a first order phase transition, see Fig. 3. The transition temperature $T_{\text {ETC }}$ is somewhat larger than $T$ at the first minimum of Fig. 2 , In view of the resemblance to walking technicolor, we call this the extended technicolor transition.

If one continues towards lower temperatures, at some $T$ the pressure must go to zero, see Fig. 4. It is zero at $\lambda_{h}=\infty$, as defined by Eq. (3.3), and since $d T / d \lambda_{h}>0$, it is negative over some range in the IR. There is a cusp in $p / T^{4}$ at the larger minimum of $T\left(\lambda_{h}\right)$ in Fig. 2 , Below this temperature, $p$ starts growing and passes zero at some $T=T_{\mathrm{TC}}$. The other stable phase is the "hadron gas" phase with $p=0$, in this strongly coupled, large $N_{c}$ theory, and the transition is the analogue of the deconfining QCD transition. The large scale separation, $T_{\mathrm{ETC}} / T_{\mathrm{TC}} \approx 600$ is due to the choice of a large value for the parameter $F$ in $(2.14)$.

A feeling of the numerical values involved, and on the general shape of $T\left(\lambda_{h}\right)$, can be obtained by adding the UV behavior $\dot{f}\left(z_{h}\right) \sim 1 / z_{h}$ and the IR limit in 2.28 resulting in

$$
\begin{aligned}
\pi T & =\frac{1}{z_{h}}+\frac{3}{2} \Xi^{2} z_{h} \\
& =\Lambda e^{1 /\left(b_{0} \lambda_{h}\right)}\left(b_{0} \lambda_{h}\right)^{b_{1} / b_{0}^{2}}+\frac{3}{2} \Xi\left(\frac{2}{3} \log \lambda_{h}\right)^{1 / 2} .
\end{aligned}
$$


Thus there is a very rapid decrease from the UV and a very slow increase in the IR. This is also reflected in the numerical value of the IR parameter $\Xi / \Lambda \sim 0.0014$ in $(2.29)$. The fit $0.00050\left(\log \lambda_{h}\right)^{0.47}$ to the curve in Fig. 2 is compatible with this value of $\Xi$. We remind that the power $1 / 2$ of $\log \lambda_{h}$ was imposed by choosing the confinement factor $\sim(\log \lambda)^{1 / 2}$ in (2.14.

\section{Spectrum of the theory: masses and quasinormal modes}

The mass spectrum is obtained as poles of a Green's function of an operator with appropriate quantum numbers in the background 2.2 with $f(z)=1$. The poles corresponding to stable particles are on the real axis; if the backround has $f(z)$ with a horizon, $f\left(z_{h}\right)=0$, the poles move to the lower complex $\omega$ plane.

To determine this Green's function in gauge/gravity duality we need the equation satisfied by fluctuations in the background $(2.2)$. The fluctuations $h_{\mu \nu}$ of the metric satisfying $\partial_{\mu} h^{\mu \nu}=$ $h_{\mu}^{\mu}=0$, i.e. the tensor fluctuations, are simple since they do not mix with other fluctuations. For dilaton fluctuations, i.e. the scalar fluctuations, one must take into account that they mix with the fluctuations of the metric. The appropriate equation has been derived in [13, 14]. Keeping first $f \neq 1$ and taking $k=0$ the equation for the gauge invariant scalar fluctuation $\phi \equiv \phi(\omega, z)$ is

$$
\ddot{\phi}+\frac{d}{d z} \log \left(f b^{3}\right) \cdot \dot{\phi}-\left(\frac{\ddot{X}}{X}+\frac{d}{d z} \log \left(f^{2} b^{3}\right) \cdot \frac{\dot{X}}{X}\right) \phi+\frac{\omega^{2}}{f^{2}} \phi=0,
$$

where $X$ was defined in (2.7). For the tensor fluctuation one simply removes the $X$-terms. For the true $T=0$ mass spectrum one takes $f=1$ and replaces $\omega^{2} \rightarrow \omega^{2}-k^{2}=m^{2}$ [15].

The methods of computing the Green's functions and their poles from 4.1) are standard [16, 17, 18, 19. To interpret the outcome it is convenient to transform (4.1) to a Schrödingerlike form with $\omega^{2}$ as the eigenvalue. In order to achieve this, one removes the $1 / f^{2}$ in 4.1 . by introducing a new variable

$$
u=\int_{0}^{z} \frac{d \bar{z}}{f(\bar{z})}
$$

which transforms the interval $0<z<z_{h}$ to $0<u<\infty$. Introducing further

$$
\psi(u)=\sqrt{b^{3}} \phi(u)
$$

the equation becomes

$$
-\psi^{\prime \prime}(u)+V_{f}^{(S)}\left(u ; z_{h}\right) \psi(u)=\omega^{2} \psi(u)
$$

where

$$
V_{f}^{(S)}\left(u ; z_{h}\right)=f^{2}\left[\frac{3 \ddot{b}}{2 b}+\frac{3 \dot{b}^{2}}{4 b^{2}}+\frac{3 \ddot{f} \dot{b}}{2 f b}+\frac{\ddot{X}}{X}+\left(\frac{3 \dot{b}}{b}+\frac{2 \dot{f}}{f}\right) \frac{\dot{X}}{X}\right]_{z=z(u)} .
$$

The superscript $S$ indicates that we are considering scalar fluctuations. For tensor fluctuation the analysis is similar, and the result for $V_{f}^{(T)}\left(u ; z_{h}\right)$ is obtained from 4.5 by simply removing 
the $X$-terms. When $u \approx z \rightarrow 0$ the requirement $b(z) \rightarrow \mathcal{L} / z$ implies that the potentials behave as

$$
V_{f}^{(i)}\left(u ; z_{h}\right) \rightarrow \frac{15}{4 u^{2}}
$$

and when $u \rightarrow \infty, z \rightarrow z_{h}$,

$$
V_{f}^{(i)}\left(u ; z_{h}\right) \rightarrow A^{(i)}\left(z_{h}\right) e^{\dot{f}_{h} u} \rightarrow 0,
$$

where

$$
A^{(i)}\left(z_{h}\right)=\left(\left.\frac{d}{d z} V_{f}^{(i)}\right|_{z \rightarrow z_{h}}\right) \lim _{z_{0} \rightarrow z_{h}}\left(z_{h}-z_{0}\right) e^{-\dot{f}_{h} u\left(z_{0}\right)} .
$$

When $f(z)=1$, the Schrödinger-type equation becomes

$$
-\psi^{\prime \prime}(z)+V_{\mathrm{Sch}}^{(i)}(z) \psi(z)=m^{2} \psi(z), \quad i=S, T
$$

where

$$
V_{\mathrm{Sch}}^{(S)}=\frac{3 \ddot{b}}{2 b}+\frac{3 \dot{b}^{2}}{4 b^{2}}+\frac{\ddot{X}}{X}+3 \frac{\dot{b}}{b} \frac{\dot{X}}{X} .
$$

For the tensor Schrödinger potential, $V_{\mathrm{Sch}}^{(T)}$, the $X$ terms are omitted. For a simple estimate of the IR behavior, one may take for $b(z)$ the numerically accurate fit $(2.29), z b(z)=\exp \left(-\Xi^{2} z^{2}\right)$ valid for large $z$. This leads to the approximate confining tensor potential

$$
V_{\mathrm{Sch}}^{(T)}=\frac{15}{4 z^{2}}+6 \Xi^{2}+9 \Xi^{4} z^{2} .
$$

This has the minimum value $(3 \sqrt{15}+6) \Xi^{2}$ at $\Xi^{2} z^{2}=\sqrt{5 / 12}$ and the mass spectrum $m_{n}^{2}=$ $12 \Xi^{2}(n+2)$. However, the existence of the dynamical ETC and TC scales produces interesting structures in between.

\section{Spectrum in a model of the deconfinement transition}

To appreciate the relation between $f=1$ and $f \neq 1$ cases in the dilaton background (2.2), we shall first consider a simplified version of the TC or deconfinement transition 20. It is based on the ansatz2

$$
b(z)=\frac{1}{z} e^{-\frac{1}{3} z^{2}}
$$

from which one can derive $\lambda(z), \beta(\lambda)$ and, in particular,

$$
f(z)=1-\frac{\left(z^{2}-1\right) e^{z^{2}}+1}{\left(z_{h}^{2}-1\right) e^{z_{h}^{2}}+1}, \quad T\left(z_{h}\right)=\frac{1}{2 \pi} \frac{z_{h}^{3}}{z_{h}^{2}-1+e^{-z_{h}^{2}}} .
$$

The tensor potential in this case is

$$
V_{\mathrm{Sch}}^{(T)}(z)=\frac{3 \ddot{b}}{2 b}+\frac{3 \dot{b}^{2}}{4 b^{2}}=\frac{15}{4 z^{2}}+2+z^{2},
$$

\footnotetext{
${ }^{2}$ For simplicity, we fix the IR scale $\Xi^{2}=1 / 3$ in this section.
} 


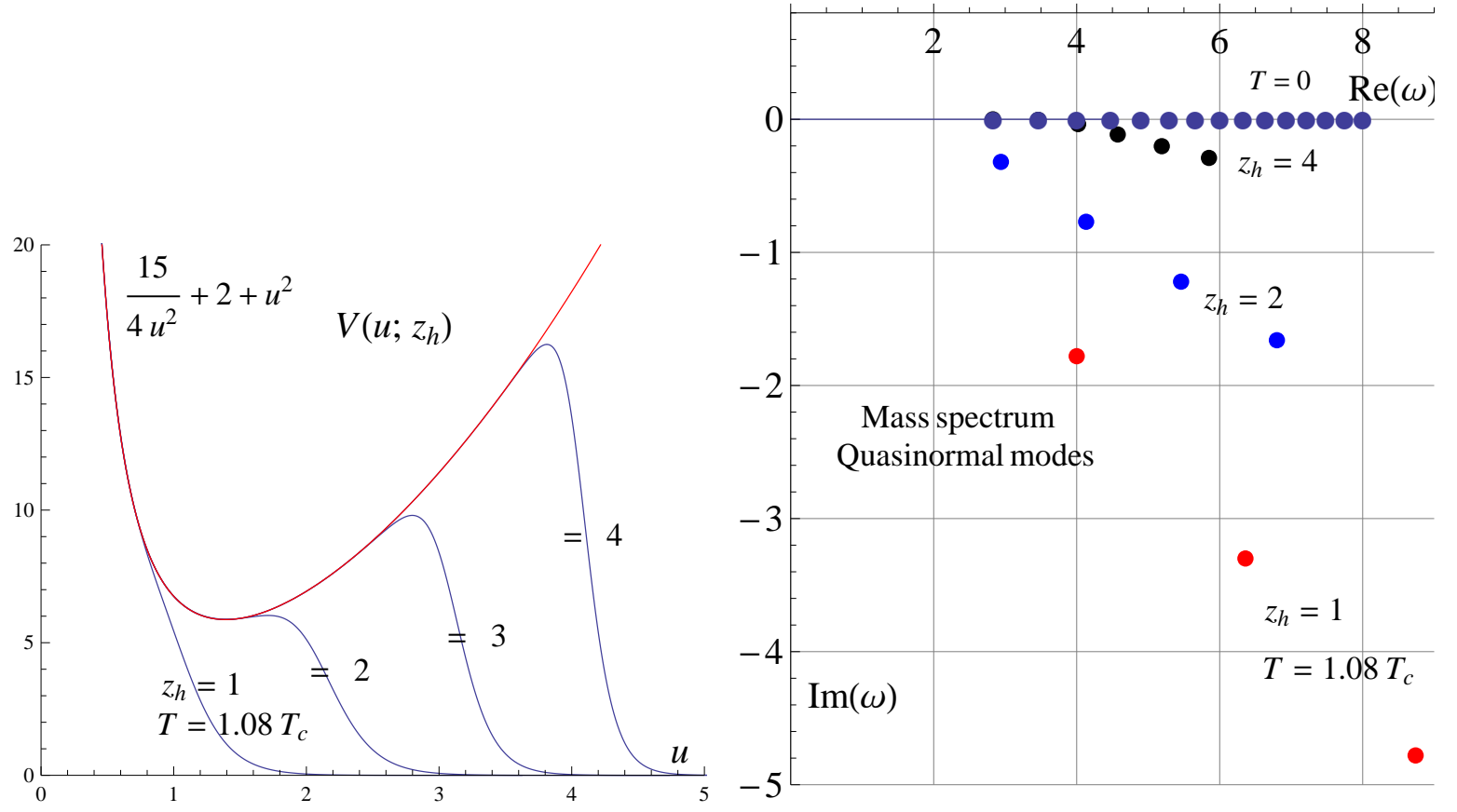

Figure 5: Left panel: The tensor potential $V_{f}^{(T)}$ given by 4.5 with $X$-terms omitted for various values of $z_{h}$. For increasing $z_{h}$ the potential $V_{f}^{(T)}$ approaches the potential $V_{\text {Sch }}^{(T)}$ in 5.3 . Right panel: The corresponding quasinormal tensor frequences computed from (4.1). The mass spectrum (5.4) is plotted on the real axis. Poles with $\operatorname{Re}(\omega) \rightarrow-\operatorname{Re} \omega$ are not shown.

and from this the tensor glueball mass spectrum becomes 12

$$
m_{n}=2 \sqrt{2+n}, \quad n=0,1,2, \ldots .
$$

The curve $T=T\left(z_{h}\right)$ has a minimum $T_{\min }=0.3862$ at $z_{h}=1.466$ and the system is in a high $T$ deconfined phase for $T>T_{c}=0.4000, z_{h}<z_{c}=1.30$. Inserting (5.1) and (5.2) to 4.10 and performing the change of variables 4.2) numerically, one finds the tensor potential plotted in Fig. 5 . The overall pattern is obvious: For small $u \approx z, f(z)=1$ and $V_{f}^{(i)}\left(u ; z_{h}\right) \approx V_{\mathrm{Sch}}^{(i)}(z)$, both for scalar $(i=S)$ and tensor $(i=T)$. The effects of $z_{h}$ start being felt and $f(z)$ starts to decrease towards $f\left(z_{h}\right)=0$ when $u \approx z$ approaches $z_{h}$. Furthermore, as $z$ approaches $z_{h}$, the variable $u$ approaches infinity and, due to the $f^{2}(z)$ factor in 4.5 , the potential $V_{f}^{(i)}\left(u \rightarrow \infty ; z_{h}\right)$ approaches zero.

The mass spectrum (5.4) can be derived by solving the Schrödinger equation (4.9). To solve the quasinormal spectrum when $f \neq 1$ it is actually most convenient to go back to (4.1) and use standard techniques for computing the Green's function. To this end one solves first Eq. (4.1) numerically by starting the integration at $z=z_{h}$ expanding for small $z-z_{h}$ so that the leading term is $\sim\left(z-z_{h}\right)^{i \omega / \dot{f}\left(z_{h}\right)}$. Secondly, one constructs analytically the two linearly independent solutions, $\phi_{u} \sim 1$ and $\phi_{n} \sim z^{4}$ near $z=0$ by expanding the solution at small $z$. Finally, the quasinormal modes are those values of $\omega=\operatorname{Re} \omega+i \operatorname{Im} \omega$ for which the solution 


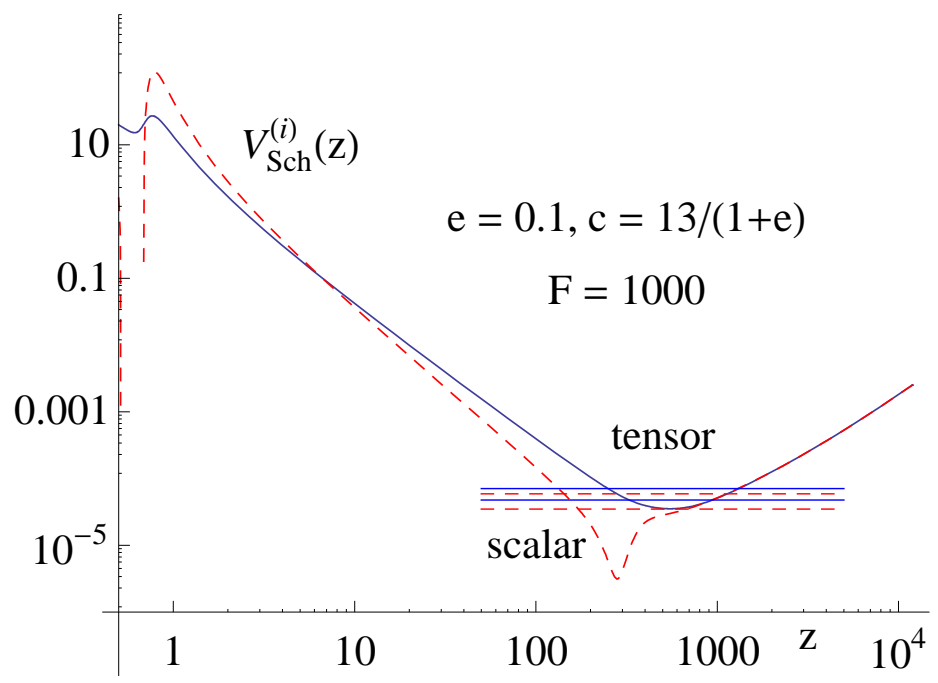

Figure 6: The scalar (dashed line) and tensor (continuous line) potentials in the IR large- $z$ TC region for $e=0.1, c=13 /(1+e), F=1000$ (note $\log _{10}$ scale). The large- $z$ tail is given by (4.11) with $\Xi \approx 0.0014 \Lambda$. Two lowest scalar and tensor excitations are plotted, the ordering is $E_{0}^{(S)}<E_{0}^{(T)}<$ $E_{1}^{(S)}<E_{1}^{(T)}$. The corresponding mass values, $m^{2} \equiv E$, are in 6.1 .

$\sim z^{4}$ at small $z$ and the coefficient of $\phi_{u}$ vanishes. Examples of the lowest quasinormal states, plotted in Fig. 5, are

$$
\begin{array}{ll}
z_{h}=1: & 4.0-1.78 i, \quad 6.36-3.3 i, \quad 8.74-4.78 i \\
z_{h}=2: & 2.94-0.32 i, \quad 4.13-0.77 i, \quad 5.46-1.22 i, \quad 6.8-1.66 i \\
z_{h}=4: & 2.83-0 . i, 3.46-0.004 i, \quad 4.02-0.036 i, \quad 4.57-0.11 i, 5.19-0.20 i, 5.85-0.29 i
\end{array}
$$

The pattern is clear: for large $z_{h}$ the quasinormal frequencies approach the real mass spectrum (5.4). However, then the system is in the unstable small black hole phase with $T^{\prime}\left(z_{h}\right)>0$. With decreasing $z_{h}$ the spectrum moves away from the real axis, and approaches $\omega=2 n( \pm 1-$ $i) / z_{h}=2 \pi \operatorname{Tn}( \pm 1-i)$ as the system enters the stable high $T$ phase. For example, $z_{h}=1$ corresponds to $T=1.08 T_{c}$, but then the states are very broad as they are melting away.

\section{Spectrum for quasi-conformal theory}

To have an overall picture of the situation, we first plot in Figs. 6 (on logarithmic scales) and 8 (linear scales, large $V_{\mathrm{Sch}}^{(S)}$ ) the $f=1$ Schrödinger potentials in 4.10 choosing the same reference values (3.1) as in the discussion of thermodynamics: $e=0.1, c=13 /(1+e)$, $F=1000$. Later we shall study the approach to an IR fixed point by taking $e \rightarrow 0$. Note that formally the potentials are plotted in units of $\Lambda, V_{\mathrm{Sch}}^{(S)} / \Lambda^{2}$ vs $\Lambda z$, but we have chosen $\Lambda=1$. 


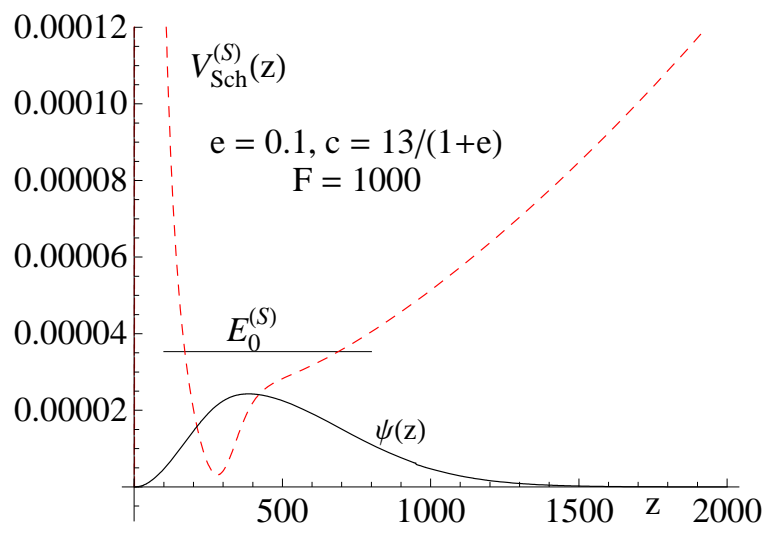

Figure 7: The scalar potential and the energy $\left(E_{0}=0.355 \cdot 10^{-4}\right)$ and wave function of the lowest scalar state (note linear scale).

Overall, the potentials follow very well the pattern in (4.11) with $\Xi \approx 1 / 730$ as in Fig. 1 . with some important modifications: there appear local minima at the ETC scale and the scalar potential has more structure than the tensor one and also binds more strongly.

\subsection{Stable states and quasinormal modes at the TC level}

The lowest energy stable states of the system are those near the minimum of the potential $V_{\text {Sch }}$. Computing the four lowest scalar and tensor stable states one finds that their mass values are (as always, in units of $\Lambda=1$ )

$$
\begin{aligned}
& m^{(S)}=(0.005948,0.0077235,0.0090700,0.010182) \\
& m^{(T)}=(0.0069018,0.0083675,0.0095586,0.010581)
\end{aligned}
$$

In spite of the prominent dip in the scalar potential the lowest scalar mass, the analogue of standard model Higgs, is thus not particularly well separated from the higher excitations. Its wave function is plotted in Fig. 7 and one sees that the narrowness of the potential well pushes the state higher. Masses should be compared with $T_{\mathrm{TC}}=1.0 \times 10^{-3} \Lambda$, see Fig. 4 , and one observes that the lowest mass $\approx 2 \pi T_{\mathrm{TC}}$.

The tensor potential is well approximated by the form (4.11). In fact, one could use this to define the IR scale $\Xi$ via the equation

$$
\Xi(c, e)=\frac{(5 / 12)^{1 / 4}}{z_{\min }}, \quad V^{\prime}\left(z_{\min }\right)=0 .
$$

Numerically, this is close to the alternative definition $(2.23), b(z) \rightarrow \exp \left(-\Xi^{2} z^{2}\right)$; for $e=$ $0.1, c(1+e)=13$ the former gives $\Xi=1 / 680$, the latter $\Xi=1 / 730$.

Since the approximation (4.11) works so well, the approximate quasinormal tensor modes can be read from the results of subsection 5 by noting that there $3 \Xi^{2}=1$, i.e., in Fig. 5 one should replace $\omega \rightarrow \sqrt{3} \Xi$. For the scalar case the quasinormal modes have not been computed. 


\subsection{Stable states and quasinormal modes at the ETC level}

The $f=1$ potential over the entire range was plotted in Fig. 6. A close-up of the ETC region, $z \sim 1, \lambda \sim 1, V_{S} \sim 1$, is shown in Fig. 8, left panel. Overall, the potential is confining but extremely wide, one has to go to $z \sim 10^{6}$ before the large $z$ part reaches the magnitude 100 of the peak in the ETC region. Due to the confining nature of the potential, there are stable states of large excitation number. In fact for the potential (4.11), which approximates very well the potential in Fig. 6, one has

$$
m_{n}^{2}=12 \Xi^{2}(n+2) .
$$

Thus, for $m=1$ the excitation number would be $n=44410$ and the mass splitting $\Delta m=$ $\sqrt{3 / n} \Xi$. However, there also are local minima in the potential. These arise due to the quasiconformal behavior of the beta function near $\lambda=1$ and the related rapid variation of the bulk fields in Fig. 1.

Potentially, the most interesting feature is that the scalar potential even dips clearly below zero, so that even stable states are thinkable. However, for all the parameters the dip seems to be so narrow that the energy levels are lifted to the metastability region.

One may ask what the basic reason for the appearance of this structure in the ETC region is. It really comes from the transition of $b(z)$ from the UV conformal region with $b(z)=\mathcal{L}_{U} / z$ to a quasi-conformal region with $b(z) \approx \mathcal{L}_{I} / z$. This is described by Eq. 2.29 and inserting this to $V_{\text {Sch }}$ one sees that the peak structure is formed.

To see the effect of minima at $V>0$, forget first the increase of the potential at very large $z$. The minima then would correspond to metastable states. To estimate their energies, one may demand that the wave function of the potential in Fig. 8 vanish at $z=0.01$ and $z=1.2$. One finds that this potential can bind two states with masses

$$
m_{0}=4.42, \quad m_{1}=8.92,
$$

shown as horizontal lines in Fig. 8. Of course, these states are only metastable. The tunneling actions for these states are of the order of 1 so that estimates of widths are are very unreliable. Restoring the increase of the potential at large $z$ one sees that these states simply disappear in the dense spectrum of large $m$ states (6.3), effectively doubling two of the states (6.3). However, the levels (6.4) will reenter when considering quasinormal modes.

The appropriate $f \neq 1$ potentials are plotted in Figs. 8 and 9 . Fig. 8 compares the $f=1$ potentials $V_{\mathrm{Sch}}^{(i)}(z)$ and $f \neq 1$ potentials $V_{f}^{(i)}\left(u ; z_{h}\right)$ at $T=T_{\mathrm{TC}}$. Fig. 9. left panel, shows the $f$ dependent potential $V_{f}^{(i)}\left(u ; z_{h}\right)$ for a wider range of $z_{h}$. To appreciate the relation $\lambda_{h}=\lambda\left(z_{h}\right)$, see figure 1. One sees that, as suggested by Fig. 5 , when $z_{h}$ approaches the UV, the metastability peak is washed away.

The quasinormal spectrum computed for various values of $z_{h}$ is also shown in Fig. 9. For large values of $z_{h}\left(\lambda_{h} \gtrsim 1\right.$ the metastability peak is efficient and there are states with negligible imaginary part. These are the metastable states suggested physically by the existence of the 

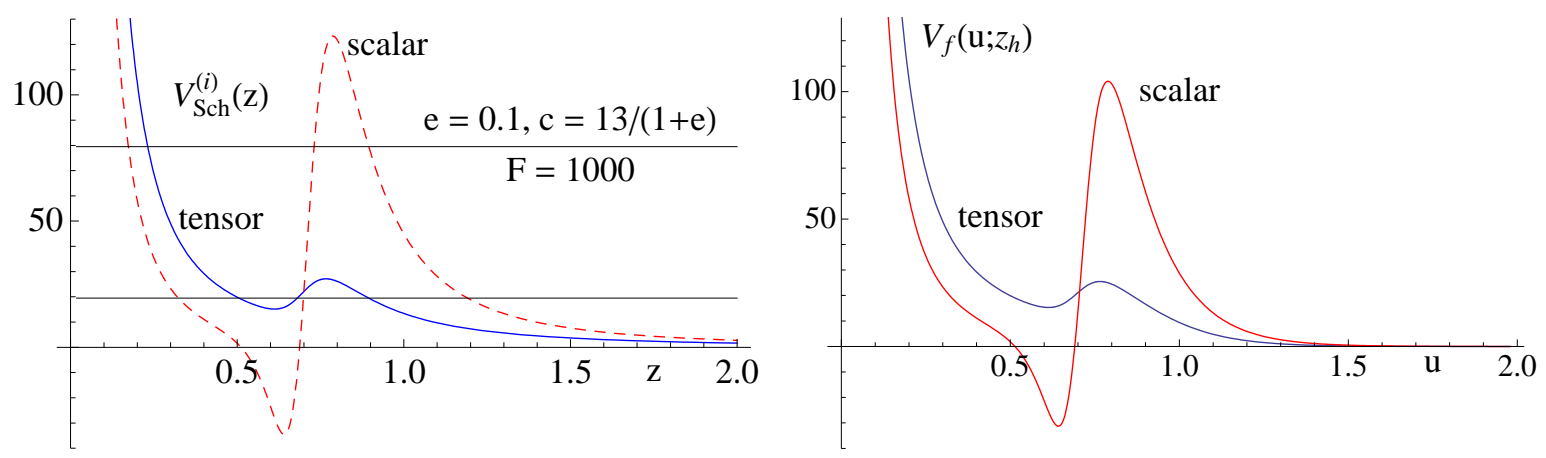

Figure 8: Left panel: The scalar and tensor potentials in the UV small- $z$ ETC region for $e=0.1$, $c=13 /(1+e), F=1000$ (note linear scale). For $z \rightarrow 0$ both potentials approach $\sim 15 /\left(4 z^{2}\right)$. For $z \rightarrow \infty$ one has to go to $z \approx 10^{6}$ before $V_{\text {Sch }}^{(i}$ ) grows back to the value 100 (Fig. 6). Right panel: The $f \neq 1$ scalar and tensor potentials $V_{f}^{(i)}\left(u ; z_{h}\right)$ in 4.5 for the same values of $e, c, F$ and for $\lambda_{h}=\lambda_{c}=0.71, z_{h}=0.71, T=T_{\mathrm{TC}}=0.6 \Lambda$.

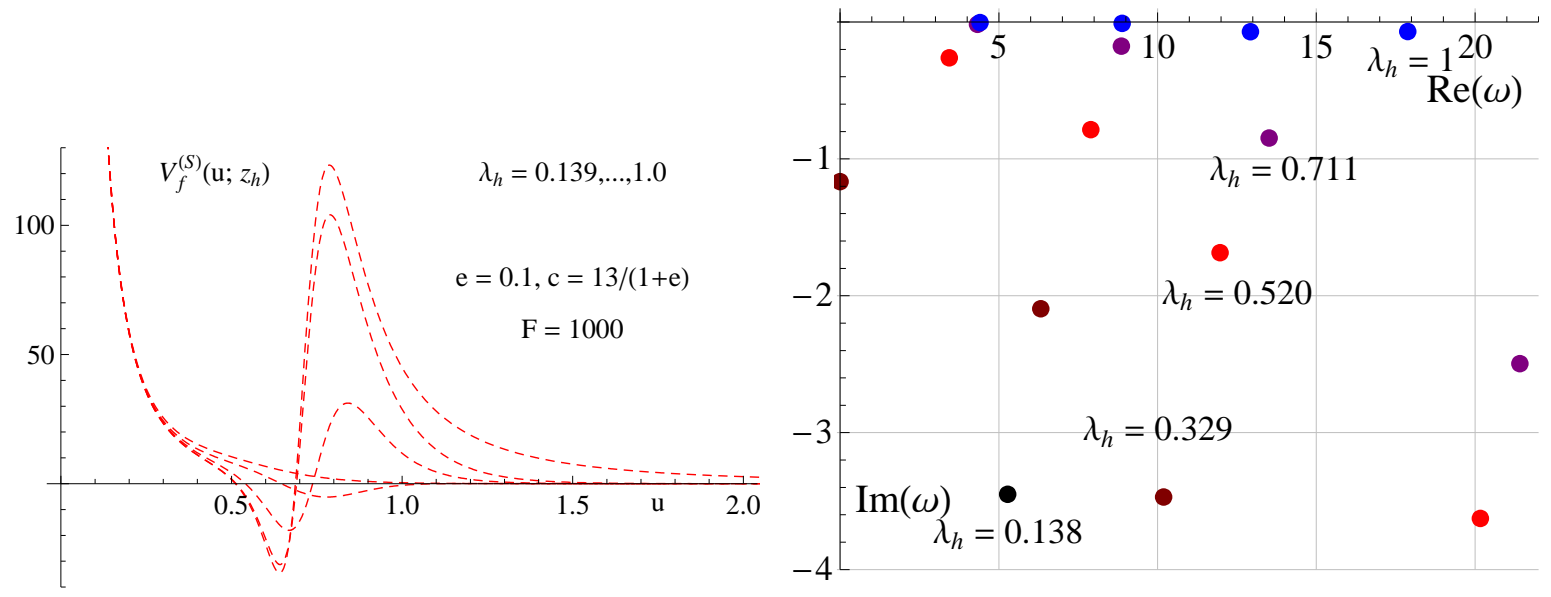

Figure 9: Left panel: The dependence of the scalar potential $V_{f}^{(S)}\left(u, z_{h}\right)$ for $\lambda_{h}=$ $0.138,0.329,0.520,0.711,1.0, z_{h}=0.57,0.719,0.724,0.92,2.96, \pi T \approx 1 / z_{h}$. The peak disappears when one is approaching the UV, when $\lambda_{h}$ decreases. Right: Corresponding quasinormal modes.

peak in the potential. Concretely, the lowest states for four of the $\lambda_{h}$ values are

$$
\begin{aligned}
& \lambda_{h}=1, z_{h}=2.96: \quad 4.41-0.0036 i, \quad 8.89-0.010 i, \quad 12.9-0.071 i, \quad 17.9-0.069 i \\
& \lambda_{h}=0.711, z_{h}=0.922: \quad 4.33-0.018 i, \quad 8.86-0.18 i, \quad 13.5-0.847 i, \quad 21.4-2.50 i \\
& \lambda_{h}=0.520, z_{h}=0.724: \quad 3.44-0.26 i, \quad 7.90-0.79 i, \quad 12.0-1.68 i, \quad 20.2-3.62 i, \\
& \lambda_{h}=0.329, z_{h}=0.719: \quad 0.00-1.17 i, \quad 6.32-2.09 i, \quad 10.2-3.47 i, \quad 29.5-4.80 i .(6.5)
\end{aligned}
$$

Comparing with (6.4) one sees that for $\lambda_{h} \gtrsim 1$ the temperature is already so low that the real parts are very close to the estimated energies of metastable states. However, now one 
also has a controlled determination of the imaginary parts. For increasing $\pi T \sim 1 / z_{h}$ the imaginary part rapidly grows, the states are melted away. The real part of the lowest state starts decreasing and soon is even very close to zero. Note that this is quite different from what was observed with the model computation plotted in Fig. 5. The reason is obvious: the pattern in Fig. 9 is determined by the effective vanishing of the ETC metastability peak when $T$ grows, while there is no such effect in the TC region.

Finally, we consider how our results are affected by the choice of the parameters in the beta function, i.e. in the dilaton potential. First, since for $e \neq 0$ the theory always confines at large $\lambda$, the large $z$ behavior and the spectra at the TC level are practically independent on the choice of parameters as long as $e \neq 0$. Second, at small $z$, i.e. at the ETC level, there arise structures more sensitively dependent on the values of the parameters. This is illustrated in Fig. 10 which gives examples of how the Schrödinger potentials vary when parameters are changed. In the plots, we show the potentials scaled with the IR scale $\Xi$ defined in $(6.2)$ vs. $\Xi z$. As we have already noted, the shape of the potential at small $z$ consists of a minimum followed by a peak as $z$ increases. There are two main effects which arise as the parameters in the potential are changed: The first effect is that the location of the high temperature minimum shifts towards larger values of $z$ as the parameter $e$ is increased. The left panel of the figure shows how this happens for two values $e=0.1$ and 0.3 . The parameter $F$ has similar effect, i.e. larger value of $F$ implies larger separation between the high and low temperature minima. The second quantitative feature is the depth of the high temperature minimum and the height of the peak. This is mainly controlled by the parameter $c$ as can be seen from the left panel of Fig. 10, and in more detail for $e=0.01$ from the right panel. In conclusion, the qualitative shape of the Schrödinger potentials and our corresponding results for the spectra appear robust as the parameters are varied so that the walking nature of the theory is preserved. Of course the quantitative results like the number of metastable states
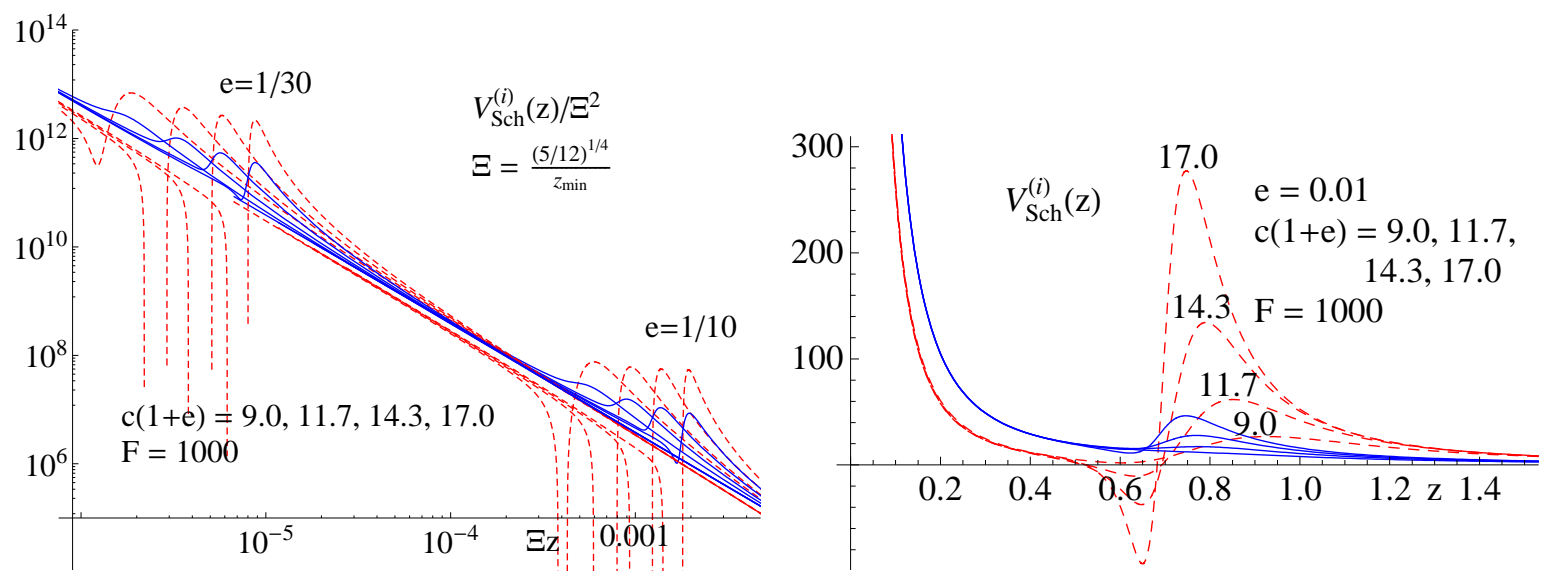

Figure 10: Left panel: The Schrödinger potentials scaled with the IR scale 6.2 for two values of $e$, plotted vs $\Xi z$. Right panel: Dependence of the Schrödinger potentials on $c$ at fixed $e=0.01$. If one increases $e$ to 0.1 , the height of the peak and the depth of the dip at $c(1+e)=14.3$ increase by $\sim 30 \%$. 
at the ETC level, and how rapidly they melt away at increasing temperature will depend on the exact numerical values chosen for the parameters.

\subsection{Approaching the IR fixed point, $e \rightarrow 0$}

When $e \rightarrow 0$ in $(2.2)$, the $\beta$ function approaches one with an IR fixed point at $\lambda=1$ (we remind that, in practice, always $\left.a=\frac{2}{3} c\right)$ :

$$
\beta(\lambda) \approx-\frac{c}{1+a}\left[(1-\lambda)^{2}+e\right] .
$$

In the limit the theory is conformal both in the UV and in the IR. Thus one expects the mass spectrum to approach zero, in the potential language the confining part $+9 \Xi^{4} z^{2}$ of the potential disappears. How this happens can be derived as follows.

Integrating $d \lambda / d t=\beta(\lambda), t=\log \left(\mu / \mu_{0}\right)$ in the approximation (6.6) one has

$$
\lambda(t)=1-\sqrt{e} \tan \left(\frac{c}{1+a} \sqrt{e} t\right),
$$

normalised so that $\lambda(0)=1$. This decreases monotonically from $+\infty$, reached at the value $t<0$ defining an IR scale:

$$
t_{\mathrm{IR}}=\log \frac{\mu_{\mathrm{IR}}}{\mu_{0}}=-\frac{(1+a) \pi}{2 c \sqrt{e}} .
$$

The UV scale can be defined as the value of $t>0$ for which $\lambda(t)$ has decreased to zero:

$$
t_{\mathrm{UV}}=\frac{(1+a) \pi}{2 c \sqrt{e}}-\frac{1+a}{c^{2}} \approx-t_{\mathrm{IR}}
$$

Thus

$$
\frac{\mu_{\mathrm{IR}}}{\mu_{\mathrm{UV}}}=e^{t_{\mathrm{IR}}-t_{\mathrm{UV}}}=\exp \left[-\left(\frac{2}{3}+\frac{1}{c}\right) \frac{\pi}{\sqrt{e}}\right],
$$

where $a=\frac{2}{3} c$ was inserted. We thus expect that, at small $e$, the IR scales and masses behave as

$$
\Xi \sim m=A \exp \left[-\frac{D}{\sqrt{e}}\right], \quad D=\left(\frac{2}{3}+\frac{1}{c}\right) \pi
$$

$A=$ constant.

This prediction works surprisingly well. All the masses are proportional to the IR scale $\Xi=(5 / 12)^{1 / 4} / z_{\min }$, defined in 6.2 . Here $z_{\min }$ is the value at which the tensor potential has its IR minimum. Its dependence on $e$ at fixed $c(1+e)$ (keeping this fixed keeps the leading UV behavior unmodified by the variation of $e$ ) is shown in Fig. 11, left. For masses the behavior is very similar:

$$
\begin{aligned}
m_{0}^{(S)}(c(1+e)=9, e) & =5.9874 \exp [-2.4348 / \sqrt{e}], \\
m_{0}^{(T)}(c(1+e)=9, e) & =6.6283 \exp [-2.4337 / \sqrt{e}], \\
m_{1}^{(S)}(c(1+e)=9, e) & =7.7630 \exp [-2.4342 / \sqrt{e}], \\
m_{0}^{(S)}(c(1+e)=17, e) & =14.671 \exp [-2.2764 / \sqrt{e}] .
\end{aligned}
$$



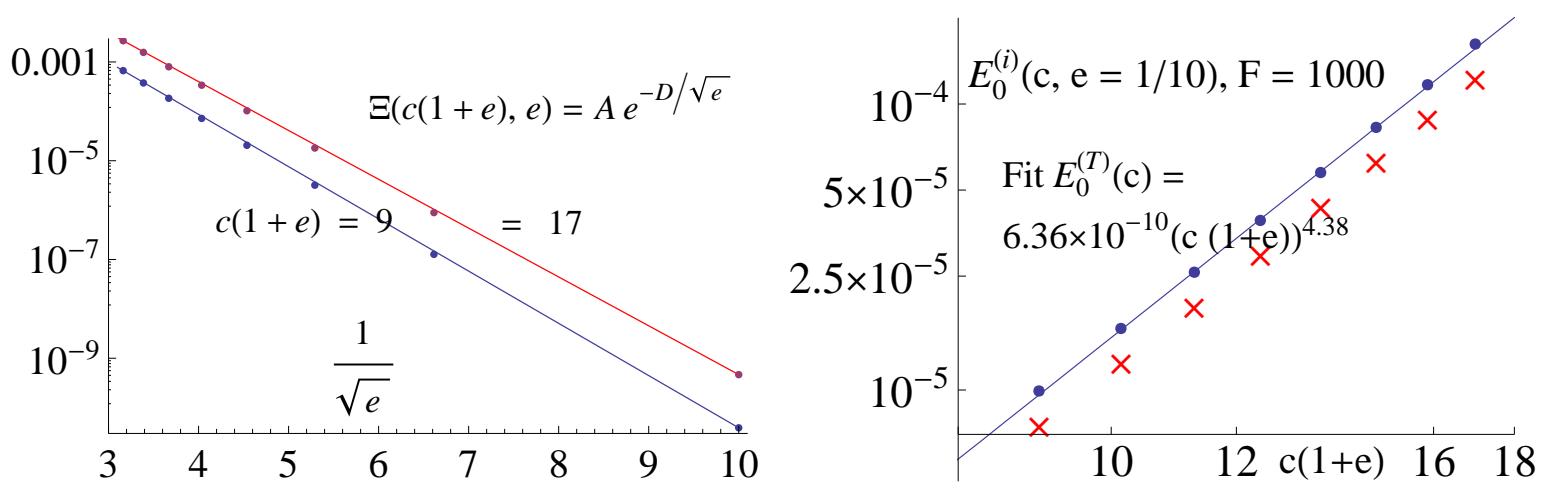

Figure 11: Left panel: The dependence of the IR scale $\Xi$ defined in Eq. 6.2 on $e$ at fixed $c(1+e)=$ $9(A=1.4769, D=2.4358)$ and $c(1+e)=17(A=3.60638, D=2.277)$. All masses behave very similarly. Right panel: The dependence of the tensor and scalar (crosses) masses squared on $c$ at fixed $e=0.1$.

According to 6.11 the calculated coefficient in the exponent is $D=7 \pi / 9=2.4435$ for $c=9$ and $D=37 \pi / 51=2.2792$ for $c=17$.

The pre-exponential factor in 1 (1.5) is non-universal. One finds that the $c$ dependence of $\Xi$ or masses at fixed $e$ is powerlike, $\sim c^{p(e)}$, with $p(e)=1.287 / e^{0.228}$ over the interval $0.01<e<0.1$. An example at fixed $e$ is shown in Fig. 11, right.

Comparing (6.12) with (6.1) one sees that the relative hierarchy of the states remains unchanged when $e \rightarrow 0$. One could have hoped the lowest scalar state to separate from the others in this limit [21], but the model clearly needs new dynamical input to accomplish this.

Since "Miransky scaling" (6.11) generally arises as one approaches a transition into a conformal theory, the prediction (6.11) and its verification by the full numerical analysis, provides another successful test for the consistency of the gauge/gravity framework considered in this paper.

\section{Conclusions}

In this paper, we have considered five dimensional dilaton gravity solutions which, in the framework of holography, are dual to a quasi-conformal gauge theory. In these theories the beta function almost vanishes, and the coupling is almost constant between two widely separated energy scales.

The dilaton potential $V(\phi)$ was determined so that using it as input to Einstein's equations, the required beta function was obtained as output. Computing numerically solutions with and without black hole, we determined the thermodynamics of these theories as well as their vacuum mass and finite temperature quasinormal spectrum. The gravity solutions imply that the corresponding field theory undergoes two phase transitions. Starting from low temperatures, there first is a transition from confining to quasi-conformal phase at $T_{\mathrm{TC}}$ and then, at significantly higher temperature $T_{\mathrm{ETC}} \sim 10^{3} T_{\mathrm{TC}}$ a further transition to a strongly interacting plasma phase. 
We determined the spectra of scalar and tensor states and studied how they are affected by quasiconformality between widely separated energy scales. The vacuum (no black hole in dual) mass spectrum is little affected, there are the usual vacuum states with masses $m^{2} \sim n T_{\mathrm{TC}}^{2}$ up to very large values of $n$. However, when approaching conformality at $e=0$, the overall scale of the mass spectrum was shown to exhibit Miransky type scaling, $m \sim \exp [-D / \sqrt{e}]$, with known $D$. This shows the robustness of the holographic scheme: the approach to conformality is built in the ansatz (1.1), but the masses are computed within a rather complicated scheme of choosing an ansatz for the bulk metric, solving the bulk fields from Einstein's equations and solving field equations in this background. Still the Miransky scaling comes out numerically with great accuracy. Another sign of the robustness is that the Schrödinger potentials computed for scalar states contain negative parts which, in principle, could predict stable states at both $T_{\mathrm{ETC}}$ and $T_{\mathrm{TC}}$. However, it seems that these dips never can bind any states.

In contrast to the vacuum spectrum, the quasinormal spectrum (black hole in the dual) in strongly affected. In fact, there are new states with small imaginary part at the $T_{\mathrm{ETC}}$ scale, see (6.5). The existence of these states is already suggested by dips in the vacuum potential. Dynamically the origin of these dips is in the transition of the system from one conformal phase in the UV $\left(b(z)=\mathcal{L}_{U} / z\right)$ to a quasi-conformal phase $\left(b(z) \approx \mathcal{L}_{I} / z\right)$.

The emphasis of this article was on quasi-conformality between two widely separated energy scales. This is what walking technicolor is built on, but it is obvious that for realistic applications to technicolor models, one would need to take better into account the dynamics of flavor degrees of freedom.

Acknowledgements. JA thanks the Magnus Ehrnrooth foundation and TA the Vaisala foundation for financial support.

\section{A Calculation of the Schrödinger potentials and their eigen- values}

In this Appendix, we shall discuss the integration of the Einstein's equations 2.16$)-(2.19)$ for the case $f=1$. As discussed in [3], there are different types of solutions (generic, bouncing), but only one special type corresponds to confinement. The trick of obtaining this solution is to start numerical integration at some very large value of $z$ or $\lambda(z)$ using as the initial condition (2.23). This automatically leads to the correct special solution. Most concretely this is seen from the large $\lambda$ limit $(2.26)$ of the beta function; the unwanted solutions have $\beta \rightarrow-3 \lambda$.

Following Eqs. (2.23) - (2.28) we start the integration of $(2.16)-(2.19)$ with $f=1$ using the initial conditions

$$
\begin{aligned}
b\left(z_{i}\right) & =b\left(\lambda_{i}\right)=b_{0} \lambda_{i}^{-2 / 3}\left(\frac{2}{3} \log \lambda_{i}\right)^{1 / 2}, \\
W\left(z_{i}\right) & =W\left(\lambda_{i}\right)=\frac{1}{3} \sqrt{V\left(\lambda_{i}\right)} \\
\lambda\left(z_{i}\right) & =\lambda_{i}
\end{aligned}
$$


where $b_{0}$ is an arbitrary constant. Now choose some numerical value for $b_{0}, z_{i}$ and $\lambda_{i}$ (say, $b_{0}=1, z_{i}=40, \lambda_{i}=10^{200}$ ), and integrate the system of differential equations towards smaller $z$ until the $b \sim 1 /\left(z-z_{U V}\right)$ divergence is encountered at the AdS boundary. Then the energy scale may be readjusted to a chosen value of $\Lambda$ (as in (2.21)) and the coordinate origin moved to $z_{U V}=0$ by the scalings described in appendix A of [4] (since $f=1$, only scalings 2 . and 3. are needed). This eliminates the effect of the arbitrarily chosen constants $b_{0}$ and $z_{i}$.

Note that since the approximation error is $\mathcal{O}(1 / \log \lambda), \lambda_{i}$ has to be very large. It also has to be very large in order to reach the large- $z$ minimum $z_{\min }$ of the potential, due to the extremely slow increase of $z(\lambda)$.

After this procedure we have a set of functions $b(z), \lambda(z)$ for each set of parameters $c, e$, $z$ ranging over the interval $\left(0, z\left(\lambda=10^{200}\right)\right)$. Given this set, the potential $V_{\text {Sch }}(z)$ can be computed from 4.10.

Once the potential has been computed, a number of lowest states of the eigenvalue spectrum can be solved using the standard shooting method. Let us choose as a reference case $c=$ $9 /(1+e), e=1 / 100$, for which $z_{\min \text {, ref }}=2.06089 \cdot 10^{10}$. We set in the reference case the boundary conditions $\psi\left(z_{l, r}\right)=0$ at $z_{l}=10^{9}$ and at $z_{r}=5 \cdot 10^{11}$, far above and below the

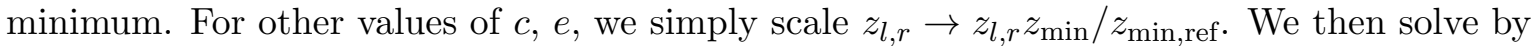
numerical rootfinding for values of $m^{2}$ where the numerically computed solutions with the two boundary conditions can be matched such that they are continuously differentiable.

\section{B Numerical evaluation of the quasinormal modes}

The quasinormal modes can be numerically evaluated from the Schrödinger type equation (4.4); for a review, see [22]. Since the equation is linear, given a value of $\omega$ a solution is completely determined by two complex constants. One of them is simply a normalization, and the other can be determined from the in-going wave condition at the horizon, which in terms of $u$ can be expressed as

$$
\psi(u) \rightarrow e^{+i \omega u} \quad \text { for large } u \text {. }
$$

This reduces the problem to finding those values of $\omega$ where the solution satisfying this condition is normalizable, i.e. $\phi(z) \rightarrow z^{4}$ when $z \approx u \rightarrow 0$.

The standard method, used in Section 5 , requires expanding the solutions around $z=0$. However, in the present gravity dual these expansions are not power series in $z$ but contain logarithmic terms, see for example (2.21). To avoid this we apply a more direct numerical method of removing the unnormalisable component from the solution.

In practice, since $\omega$ is never numerically exactly at a quasinormal mode, we expect that the solution will contain some part of the non-normalizable $\phi \rightarrow 1$ solution at the boundary, and $\psi(u) \rightarrow u^{-3 / 2}$ will therefore diverge. If we are close to a quasinormal mode, it seems intuitive that this divergence will happen only when $u$ is very small (this is the heuristic of the algorithm, since we do not have a proof of this). This suggests a way to look for the quasinormal modes: given a trial $\omega$, compute numerically the solution from large $u$ towards the boundary and find the smallest- $u$ local minimum of $\mid(\psi(u) \mid$. Denoting its position by $u_{\min }$, the zeros of $u_{\min }(\omega)$ are at the quasinormal modes. With finite precision numerics we 
can then simply look for the minima of $u_{\min }(\omega)$. See Fig. B for a visualization of the process when applied to the model of Section 5. for which the usual power expansion method could be applied.

To start the integration of 4.4 one may note Eq. 4.7), at large $u$

$$
V_{f}^{(i)}\left(u ; z_{h}\right) \rightarrow A^{(i)}\left(z_{h}\right) e^{\dot{f}_{h} u} \rightarrow 0,
$$

where $A^{(i)}\left(z_{h}\right)$ is as defined in Eq. 4.8 . With this potential 4.4$)$ has the analytic solution

$$
\psi(u)=C_{1} I_{\nu}\left(\frac{2}{-\dot{f}_{h}} \sqrt{A^{(i)}\left(z_{h}\right)} e^{\frac{1}{2} \dot{f}_{h} u}\right)+C_{2} I_{-\nu}\left(\frac{2}{-\dot{f}_{h}} \sqrt{A^{(i)}\left(z_{h}\right)} e^{\frac{1}{2} \dot{f}_{h} u}\right), \quad \nu=\frac{2 i \omega}{\dot{f}_{h}} .
$$

where the first term has the correct large $u$ limit $e^{+i \omega u}$. One can then use this evaluated at some $u$ somewhat larger than $z_{h}$ as the initial condition of numerical integration. This allows us to choose the solution with the correct asymptotic at infinity, which is the first term, and use the solution for the exponential potential to start the numerics at a somewhat smaller $\mathrm{u}$. With this improvement, the algorithm works in the analytic model of Section 5 for $z_{h}=1 \ldots 4$, although at $z_{h}=1$ there still seems to be a tendency for the minima to lie at a too small $\operatorname{Im} \omega$.
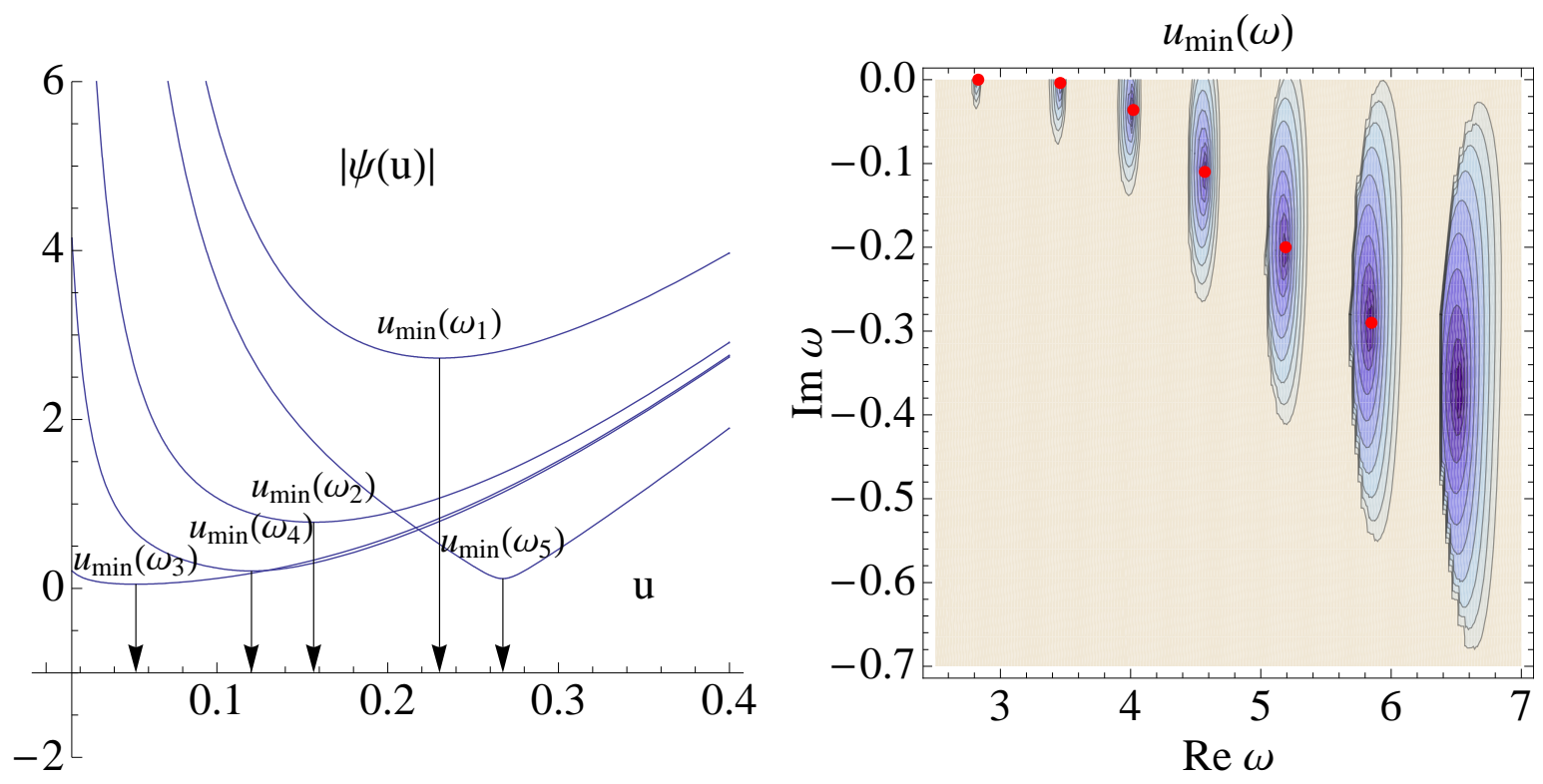

Figure 12: The numerical method applied to the analytic model (5.1) with $z_{h}=4$. Left panel: An example of looking for the fourth minimum. The plot shows $|\psi(u ; \omega)|$ for $\omega_{n} \in\{4.550-0.1000 i, 4.573-$ $0.1083 i, 4.575-0.1137 i, 4.576-0.1125 i, 4.597-0.1167 i\}$. The arrows mark the location of the minima $u_{\min }(\omega)$. Notice how the minima first approach the origin, then after the closest point $\omega_{3}=4.575-$ $0.1137 i$ they again start to move away. This is the computed value of the quasinormal mode. Right panel: The minima $u_{\min }(\omega)$ on the complex plane. On the gray area $u_{\min }>0.5$, and $u_{\min }$ decreases for shades towards dark blue. The red dots show the positions calculated in fig. 5 for comparison. 


\section{References}

[1] For a review, see F. Sannino, "Conformal Dynamics for TeV Physics and Cosmology," arXiv:0911.0931 [hep-ph].

[2] U. Gursoy, E. Kiritsis and F. Nitti, "Exploring improved holographic theories for QCD: Part II," JHEP 0802, 019 (2008) arXiv:0707.1349 [hep-th]].

[3] U. Gursoy, E. Kiritsis, L. Mazzanti and F. Nitti, "Holography and Thermodynamics of 5D Dilaton-gravity," JHEP 0905, 033 (2009) [arXiv:0812.0792 [hep-th]].

[4] U. Gursoy, E. Kiritsis, L. Mazzanti and F. Nitti, "Improved Holographic Yang-Mills at Finite Temperature: Comparison with Data," Nucl. Phys. B 820, 148 (2009) arXiv:0903.2859 [hep-th]].

[5] J. Alanen, K. Kajantie and V. Suur-Uski, "A gauge/gravity duality model for gauge theory thermodynamics," Phys. Rev. D 80, 126008 (2009) arXiv:0911.2114 [hep-ph]].

[6] J. Alanen, K. Kajantie and K. Tuominen, "Thermodynamics of Quasi Conformal Theories From Gauge/Gravity Duality," Phys. Rev. D 82, 055024 (2010) arXiv:1003.5499 [hep-ph]].

[7] J. Alanen and K. Kajantie, "Thermodynamics of a field theory with infrared fixed point from gauge/gravity duality," Phys. Rev. D, 81, 046003 (2010) arXiv:0912.4128 [hep-ph].

[8] M. Kaminski, K. Landsteiner, F. Pena-Benitez, J. Erdmenger, C. Greubel and P. Kerner, "Quasinormal modes of massive charged flavor branes," JHEP 1003, 117 (2010) arXiv:0911.3544 [hep-th]].

[9] D. B. Kaplan, J. W. Lee, D. T. Son and M. A. Stephanov, "Conformality Lost," Phys. Rev. D 80 (2009) 125005 arXiv:0905.4752 [hep-th]].

[10] C. Nunez, I. Papadimitriou and M. Piai, "Walking Dynamics from String Duals," arXiv:0812.3655 [hep-th].

[11] D. Elander, C. Nunez and M. Piai, "A light scalar from walking solutions in gauge-string duality," Phys. Lett. B 686, 64 (2010) arXiv:0908.2808 [hep-th]].

[12] A. Karch, E. Katz, D. T. Son and M. A. Stephanov, "Linear Confinement and AdS/QCD," Phys. Rev. D 74, 015005 (2006) arXiv:hep-ph/0602229.

[13] T. Springer, "Sound Mode Hydrodynamics from Bulk Scalar Fields," Phys. Rev. D79, 046003 (2009). [arXiv:0810.4354 [hep-th]].

[14] T. Springer, C. Gale, S. Jeon, "Bulk spectral functions in single and multi-scalar gravity duals," Phys. Rev. D82, 126011 (2010). arXiv:1010.2760 [hep-th]].

[15] E. Kiritsis and F. Nitti, "On massless 4D Gravitons from 5D Asymptotically AdS Spacetimes," Nucl. Phys. B 772, 67 (2007) arXiv:hep-th/0611344. 
[16] G. Policastro and A. Starinets, "On the absorption by near-extremal black branes," Nucl. Phys. B 610, 117 (2001) arXiv:hep-th/0104065].

[17] D. T. Son and A. O. Starinets, "Minkowski-space correlators in AdS/CFT correspondence: Recipe and applications," JHEP 0209, 042 (2002) arXiv:hep-th/0205051.

[18] P. K. Kovtun and A. O. Starinets, "Quasinormal modes and holography," Phys. Rev. D 72, 086009 (2005) arXiv:hep-th/0506184.

[19] P. Kovtun and A. Starinets, "Thermal spectral functions of strongly coupled N = 4 supersymmetric Yang-Mills theory," Phys. Rev. Lett. 96, 131601 (2006) arXiv:hepth/0602059.

[20] K. Kajantie, M. Krssak, M. Vepsalainen, A. Vuorinen, "Frequency and wave number dependence of the shear correlator in strongly coupled hot Yang-Mills theory," arXiv:1104.5352 [hep-ph]].

[21] D. D. Dietrich, F. Sannino and K. Tuominen, "Light composite Higgs from higher representations versus electroweak Phys. Rev. D 72, 055001 (2005) arXiv:hep-ph/0505059.

[22] E. Berti, V. Cardoso and A. O. Starinets, "Quasinormal modes of black holes and black branes," Class. Quant. Grav. 26, 163001 (2009) arXiv:0905.2975 [gr-qc]]. 